Chronisch entzündliche Darmerkrankungen

\title{
Biosimilar ermöglicht mehr Patienten eine moderne Behandlung
}

\begin{abstract}
"Ziel der Therapie bei Patienten mit einer chronisch entzündlichen Darmerkrankung (CED) ist die rasche Induktion und langfristige Erhaltung einer klinischen Remission, um Komplikationen und insbesondere operative Eingriffe zu verhindern“, erläuterte Privatdozent Dr. Bernd Bokemeyer, Minden. Ein wichtiges und sehr effektives Therapiekonzept dabei seien die TNF- $\alpha$-Inhibitoren wie Infliximab. Doch viele Betroffene erhielten diese Therapie nicht, wobei Angst vor Nebenwirkungen aber auch die Kosten eine wichtige Rolle spielen. In Deutschland werden $>10 \%$ der CED-Patienten mit einem Biologikum behandelt. „Moderne Biologika sind aber unverzichtbar, um eine mukosale Heilung zu erreichen“, so Bokemeyer. Biosimilars hätten in den letzten Jahren
\end{abstract}

dazu beigetragen, die Versorgungslücke zu schließen. „Sie tragen durch ihre Wirtschaftlichkeit dazu bei, dass mehr CED-Patienten der Zugang zu einer Biologikatherapie ermöglicht wird“.

\section{Kein signifikanter Unterschied zum Originator}

Seit vier Jahren steht als preiswerte Alternative zum originalen TNF- $\alpha$-Inhibitor mit Infliximab CT-P13 (Inflectra ${ }^{\mathrm{TM}}$ ) ein Biosimliar zur Verfügung. Bisher wurden damit Erfahrungen aus $>100.000$ Patientenjahren gesammelt und die Wirksamkeit bei 7.000 Patienten in $>50$ Studien belegt. In einer randomisierten, doppelblinden 54-wöchigen Studie bei 214 CrohnPatienten ergaben sich nach sechs Wochen keine signifikanten Unterschiede.
Die klinische Ansprechrate lag unter Infliximab CT-P13 bei 71,4\% (Originatorprodukt: 75,2\%). Auch nach 30 Wochen erwiesen sich die beiden Präparate als gleich wirksam (Ansprechrate CT-P13 $75,7 \%$ vs. Originator 75,2\%). Was das Erreichen der Remission betrifft, so schnitten auch hier beide Präparate gleich gut ab (55,0 vs. 56,9\%) [KIM YH et al. ECCO 2017, DOP061, AOCC 2017, PO48, \#102].

Eine optimale Therapie mit einem TNF- $\alpha$-Inhibitor erfordert vor allem bei unzureichendem Ansprechen die Bestimmung der Talspiegel. Bisher dauerte es mehrere Tage bis Wochen, bis solche Laborergebnisse vorlagen. „Mit einem neuen Point-of-care-Test kann jetzt sofort der Infliximab-Talspiegel bestimmt werden, sodass notwendige Therapieanpassungen auch unmittelbar eingeleitet werden können“, betonte Dr. Sascha Gotthardt, Pfizer, Berlin. Dr. med. Peter Stiefelhagen

Pressegespräch „Bridging the Gap - Wege zur Optimierung der CED-Therapie“, Viszeralmedizin 2017, 15.9.2017, Dresden; Veranstalter: Pfizer

\section{Lage des Primärtumors entscheidend für die Therapiewahl}

Darmtumoren sind eine biologisch heterogene Gruppe. Die molekularen und phänotypischen Charakteristika variieren in Abhängigkeit von der Primärlokalisation des Tumors, erklärte Professor Dirk Arnold, Hamburg. „Die Differenzierung zwischen links- und rechtsseitigen Tumoren ist bei Patienten mit metastasiertem Kolorelktalkarzinom (mCRC) auch klinisch relevant. So haben mCRCPatienten mit RAS-Wildtyp-Tumor, deren Primarius im rechtsseitigen Kolon lokalisiert ist, eine schlechtere Prognose als Patienten, deren Primärtumor distal der splenischen Flexur gelegen ist“, so der Onkologe [Arnold D et al. Ann Oncol 2017; 28: $1713-29$ ].

\section{Primäre Tumorlokalisation} entscheidet über Therapiewahl

Retrospektive Subgruppenanalysen verschiedener klinischer Studien konnten für mCRC-Patienten mit RAS-Wildtyp und rechtsseitigem Primarius zeigen, dass diese von einer Primärtherapie mit dem Angiogenesehemmer Bevacizumab (Avastin $^{\circledR}$ ) in Kombination mit einer Chemotherapie profitieren. „Dagegen ist nach derzeitigem Kenntnisstand kein Nutzen eines EGFR(Epidermal Growth Factor Receptor)-Antikörpers gegenüber einer Chemotherapie erkennbar", berichtete Arnold. Die Arbeitsgemeinschaft Internistische Onkologie (AIO) empfehle daher für Patienten mit rechtsseitigem RAS-Wildtyp-Tumor eine Bevacizumabhaltige Therapie [www.aio-portal.de/tl_ files/aio/stellungnahmen/Statement $\% 20$ der\%20AIO_update\%20Oktober\%20 2016.pdf].

Daten, die im Rahmen der Jahrestagung der American Society of Clinical Oncology (ASCO) 2017 vorgestellt wurden, belegen, dass der BRAF-Status bei mCRC-Patienten unabhängig von der Tumorprimärlokalisation ein starker ne- gativer prognostischer Faktor ist [3]. Anders ausgedrückt hängt die primäre Tumorlokalisation als Surrogatparameter für die Prognose sowie für einen numerischen Überlebensvorteil unter Bevacizumab - und damit für die Therapiewahl - nicht von einer BRAF-Mutation ab [Innocenti F et al. J Clin Oncol 2017; 35 (Suppl):\#3504].

\section{Therapieoption nach Progress}

Wie Arnold ergänzend hinzufügte, scheint die Kombination aus Bevacizumab und einer Chemotherapie bei RAS-Wildtyp-Patienten mit rechtsseitiger Tumorlokalisation nicht zuletzt deshalb vorteilhaft zu sein, weil die Antiangiogenese mit dem VEGF(Vascular Endothelial Growth Factor)-Antikörper auch nach der Progression in der Zweitlinie fortgesetzt werden kann - bei gleichzeitigem Wechsel der Chemotherapie. „Kombinationen mit weiteren Antikörpern können dann ab der dritten Linie eingesetzt werden“, erläuterte Arnold.

Dr. Silke Wedekind

Pressegespräch „First-Line-Therapie \& beyond: Einsatz von Avastin ${ }^{\circledast}$ beim mCRC", Frankfurt/M., 25.7.2017; Veranstalter: Roche Pharma. 